\title{
Cardiac Disease and Diabetes Mellitus: Correlated?
}

\section{Rabindra Nath Das}

Department of Statistics, University of Burdwan, West Bengal, India

*Corresponding author: Rabindra Nath Das, Department of Statistics, University of Burdwan, West Bengal, India, Tel: +91-9232638970; Email: rabin.bwn@gmail.com

Received date: September 26, 2017; Accepted date: September 28, 2017; Published date: September 30, 2017

Copyright: $\odot 2017$ Das RN. This is an open access article distributed under the terms of the Creative Commons Attribution License, which permits unrestricted use, distribution, and reproduction in any medium, provided the original author and source are credited.

\begin{abstract}
It is observed in practice that most of the diabetes patients have cardiovascular disease. Researchers and medical practitioners try to locate the hidden association between the cardiovascular disease and diabetes mellitus. But both the characters are attribute. It is not easy to derive the association between two attribute characters when many other variables/attribute characters are simultaneously influencing on them. The current report have suggested some approaches to examine the association between the cardiovascular disease and diabetes mellitus. Based on the suggested approaches, the report has examined the association between the two diseases using a real data set. The report has concluded that these two diseases are correlated.
\end{abstract}

Keywords: Cardiovascular disease; Diabetes mellitus; Disease marker; Haemoglobin A1c; Joint mean variance models; Total cholesterol

\section{Correlation between Cardiovascular Disease and Diabetes Mellitus}

Many research articles have pointed that diabetes mellitus (DM) and cardiac disease are strongly correlated, and practically they exist together [1-4]. Framingham Heart Study first pointed over 37 years ago that an increased risk of congestive heart failure was observed in diabetes patients [5]. Is there any relationship between DM and cardiovascular disease (CVD)? How do we examine the relationship between DM and CVD? Which one does occur first? To examine the correlation between $\mathrm{DM}$ and CVD, we have to examine the relationship between the diabetes markers and cardiac disease markers. There are four well-known diabetes markers [6]. According to American Diabetes Association (ADA 2013), the diabetes markers are Haemoglobin Alc $(\mathrm{HbAlc} \geq 7.0 \mathrm{mmol} / \mathrm{L})$, 2-hour plasma $(\geq 11.1$ $\mathrm{mmol} / \mathrm{L}$ ) during an oral glucose tolerance test, fasting (no caloric intake for at least 8 hours) plasma glucose (FPG $\geq 7.0 \mathrm{mmol} / \mathrm{L}$ ), and random plasma glucose $(\geq 11.1 \mathrm{mmol} / \mathrm{L})$. On the other hand there is no well-known cardiovascular disease marker. Practically, total cholesterol (TC) is considered as a CVD marker. It is known that TC is the total of low-density lipoprotein (LDL-C), very low-density lipoprotein (VLDL-C), and high-density lipoprotein (HDL-C). Note that Triglycerides is not included in TC, and the normal range of TC is below $200(\mathrm{mg} / \mathrm{dL})$.

The correlation or association between DM and CVD may be examined in the following ways.

\section{Approach 1}

On examining the association between a diabetes marker with TC, its components, cardiac parameters (systolic blood pressure (BP), diastolic $\mathrm{BP}$, maximum $\mathrm{BP}$, basal $\mathrm{BP}$, mean central venous pressure, mean arterial $\mathrm{BP}$, basal heart rate (HR), peak HR, maximum HR, ejection fraction, etc), cardiac disease status (hypertension, stroke, recent angioplasty, new myocardial infraction (MI), recent bypass surgery etc.), cardiac disease history (history of hypertension, coronary artery bypass surgery, MI, angioplasty etc.), and along with the relevant explanatory variables.

\section{Approach 2}

On examining the association between TC or its component with the remaining other explanatory variables along with DM markers, and history of DM.

\section{Approach 3}

On examining the association between a cardiac parameter with the DM markers, history of DM, and along with the remaining other explanatory variables.

These associations can be examined only by an appropriate stochastic or statistical modeling of the targeted response variable with the interested explanatory variables. Simple correlation or partial correlation is not suitable for examining these associations. To study the association between DM and CVD, an appropriate data set should contain all diabetes markers, TC and its all components, cardiac parameters, cardiac disease status, history of cardiac disease or diabetic disease, and along with all relevant explanatory variables. It is very rare to have such a data set in the previous research articles.

To illustrate the above three approaches for examining the association between DM and CVD, we have consider a data set from [1]. A short description of the data set is reproduced herein. It contains 366 subjects with 20 explanatory variables/factors, which are sex (male=1, female=2), age, weight, height, stabilized glucose (STB.GL), glycosolated hemoglobin (GLYHB or HbA1c), total cholesterol (TC), high density lipoprotein (HDL-C), lipid ratio=cholesterol/HDL-C (Ratio), first systolic blood pressure (BP) (BP.1s), first diastolic BP (BP. 1d), body mass index (BMI), waist measurement (Waist), hip measurement (Hip), index of fat distribution is equal to waist/hips ratio (IFD), second systolic BP (BP.2s), second diastolic BP (BP.2d), frame $\quad(1=$ small, $\quad 2=$ medium, $\quad 3=$ large $) \quad$ (Frame), location 
(1=Buckingham, 2=Louisa) (Location), postprandial time when labs were drawn (TIM.PN).

The above data set contains only one DM marker, namely glycosolated hemoglobin (GLYHB or HbAlc). It contains TC, HDL-C and lipid ratio. It contains two cardiac parameters, namely first systolic blood pressure (BP) (BP.1s), and first diastolic BP (BP.1d). Analysis results of $\mathrm{HbA} 1 \mathrm{c}$ and $\mathrm{TC}$ are given in [1]. We have consider these two analyses to describe the above three approaches. In addition, we have derived the results in the report based on addition analyses of HDL-C, lipid ratio, first systolic blood pressure (BP) (BP.1s), and first diastolic BP (BP.1d) to illustrate the above three approaches.

\section{Approach 1}

On examining the association between a diabetes marker with TC, its components, cardiac parameters, cardiac disease status and history

In the above data set, $\mathrm{HbAlc}$ is only one diabetes marker which has been modelled using the remaining other variables. It shows that mean $\mathrm{HbAlc}$ is positively correlated with $\mathrm{TC}(\mathrm{P}=0.002)$, implying that $\mathrm{HbAlc}$ is high or low according as TC increases or decreases. In addition, mean value of $\mathrm{HbAlc}$ is positively correlated with HDL-C $(\mathrm{P}=0.082)$, implying that $\mathrm{HbA1c}$ increases or decreases according as HDL-C is high or low. The mean value of HbAlc is reciprocally correlated with the interaction effect of HDL-C and TC, that is (HDL$\left.\mathrm{C}^{\star} \mathrm{TC}\right)(\mathrm{P}=0.032)$, implying that $\mathrm{HbAlc}$ increases as the interaction effect (HDL-C ${ }^{\star} \mathrm{TC}$ ) decreases. Note that $\mathrm{HbAlc}$ is not associated with the cardiac parameters BP.1s and BP.1d. The detailed results are given in [1].

\section{Approach 2}

On examining the association between TC or its component with the remaining other explanatory variables along with DM markers, and history of DM.

\section{Analysis of TC}

In the above data set, total cholesterol (TC), high density lipoprotein (HDL-C), lipid ratio=cholesterol/HDL-C (Ratio) are three cardiovascular disease markers. It shows that mean value of TC is positively correlated with $\mathrm{HbAlc}(\mathrm{P}=0.053)$, implying that $\mathrm{TC}$ is high or low according as $\mathrm{HbAlc}$ increases or decreases.

\section{Analysis of HDL-C}

Analysis of HDL-C is not given in [1]. We have performed the analysis (joint Log-normal generalized linear models) of HDL-C. It is observed that HDL-C is not associated with glycosolated hemoglobin (HbAlc), but the mean value of HDL-C is positively correlated with the stabilized glucose $(\mathrm{P}=0.033)$, implying that HDL-C increases as the stabilized glucose increases. Also the variance of HDL-C is positively correlated with the stabilized glucose $(\mathrm{P}<0.001)$, implying that variance of HDL-C increases as the stabilized glucose increases.

\section{Analysis of lipid ratio (TC/HDL-C)}

Analysis of lipid ratio (TC/HDL-C) is not given in [1]. We have performed the analysis (joint Log-normal generalized linear models) of lipid ratio. It is observed that mean lipid ratio is positively partially correlated with the stabilized glucose $(\mathrm{P}=0.142)$, implying that the lipid ratio increases as the stabilized glucose increases. In addition, mean lipid ratio is negatively partially correlated with $\mathrm{HbAlc}(\mathrm{P}=0.117)$, implying that the lipid ratio increases as the HbAlc decreases. The variance of lipid ratio is positively correlated with the stabilized glucose $(\mathrm{P}<0.001)$, implying that the variance of lipid ratio increases as the stabilized glucose increases. Again, the variance of lipid ratio is inversely correlated with the $\mathrm{HbAlc}(\mathrm{P}=0.049)$, implying that the variance of lipid ratio increases as the HbAlc decreases.

\section{Approach 3}

On examining the association between a cardiac parameter with the DM markers, history of DM, and along with the remaining other explanatory variables.

In the above data set, first systolic blood pressure (BP) (BP.1s) and first diastolic BP (BP.1d) are only two cardiac parameters. Analysis of first systolic blood pressure is not given in [1]. We have performed the analysis (joint Log-normal generalized linear models) of first systolic BP. It is observed that mean systolic BP is positively partially correlated with the stabilized glucose (0.184), indicating that the systolic BP increases as the stabilized glucose increases. Moreover, mean systolic $\mathrm{BP}$ is positively partially correlated with the HbAlc $(\mathrm{P}=0.097)$, implying that the systolic BP increases as the HbAlc increases. The variance of first systolic $\mathrm{BP}$ is negatively correlated with the stabilized glucose $(\mathrm{P}=0.015)$, indicating that the systolic $\mathrm{BP}$ variance increases as the stabilized glucose decreases. Also the variance of first systolic BP is positively partially correlated with the $\mathrm{HbAlc}(\mathrm{P}=0.239)$, indicating that the systolic BP variance increases as the HbAlc increases. Note that the first diastolic BP is not associated with the HbAlc and the stabilized glucose. The detailed analyses of HDL-C, lipid ratio, systolic BP and diastolic BP are not given here. We will submit these results in our subsequent full research paper. One can verify our comments from the data as stated above.

The above data set has many demerits. It contains only one diabetes marker (HbAlc). It should contain the entire diabetes marker, and the history of diabetes of the patients. The data set does not contain LDLC, VLDL-C, many cardiac parameters, cardiac disease status, and history of cardiac disease. The considered data set contains a few of the explanatory covariates. With this data set we have explained all the proposed approaches to examine the correlation between DM and CVD. The data set has been analyzed using both the joint generalized liner Log-normal and gamma models [7-11].

For the considered data set, it has been established that the diabetes marker $\mathrm{HbAlc}$ is correlated with total cholesterol, HDL-C, and the interaction effect of HDL-C and TC (Approach 1). Analysis of TC shows that TC is correlated with HbAlc. Analysis of HDL-C shows that HDL-C is correlated with the stabilized glucose. Analysis of lipid ratio (TC/HDL-C) shows that the lipid ratio is correlated with the stabilized glucose and HbAlc. Approach 3 shows that systolic BP is correlated with the stabilized glucose and HbA1c. Thus, the three suggested approaches have shown that the diabetes mellitus and cardiovascular diseases are correlated. The report may help the future researchers to study the inter-relationship between the diabetes mellitus and cardiovascular diseases.

\section{References}

1. Das RN (2016) Relationship between diabetes mellitus and coronary heart disease. Curr Diabetes Rev 12: 1-12.

2. Huxley R, Barzi F, Woodward M (2006) Excess risk of fatal coronary heart disease associated with diabetes in men and women: Meta-analysis of 37 prospective cohort studies. BMJ 332: 73-78. 
3. Das RN (2017) Association between diabetes markers and cholesterol. Diabetes Manag 7: 247-249.

4. Das RN (2016) Diabetes mellitus \& cardiovascular disease: Co-existence? BAOJ Diabet 2: 1-10.

5. Kannel WB, McGee DL (1979) Diabetes and cardiovascular disease, The Framingham study. JAMA 241: 2035-2038.

6. UKPDS Group (2001) The UKPDS risk engine: A model for the risk of coronary heart disease in type II diabetes (UKPDS 56). Clin Sci 101: 671-679.

7. Lee Y, Nelder JA, Pawitan Y (2006) Generalized linear models with random effects: Unified analysis via H-likelihood, Chapman \& Hall, London.
8. Das RN, Lee Y (2009) Log normal versus gamma models for analyzing data from quality-improvement experiments. Quality Engineering 21: 79-87.

9. Das RN, Park JS (2012) Discrepancy in regression estimates between lognormal and gamma: Some case studies. J App Stat 39: 97-111.

10. Firth D (1988) Multiplicative errors: Log-normal or gamma? J R Stat Soc Series B Stat Methodol 50: 266-268.

11. Das RN (2014) Robust response surfaces, regression, and positive data analyses. Chapman \& Hall, London. 